Research Paper

\title{
Genipin increases oxaliplatin-induced cell death through autophagy in gastric cancer
}

\author{
Bo Ram Kim ${ }^{1 *}$, Yoon A Jeong 2 , Dae Young Kim², Jung Lim Kim¹, Soyeon Jeong1, Yoo Jin $\mathrm{Na}^{2}$, Hye Kyeong

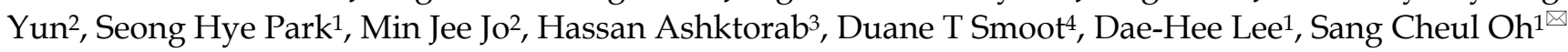 \\ 1. Department of Oncology, Korea University Guro Hospital, Korea University College of Medicine, Seoul, Republic of Korea. \\ 2. Graduate School of Medicine, Korea University College of Medicine, Seoul, Republic of Korea. \\ 3. Department of Medicine, Howard University, Washington, District of Columbia, 20060, USA. \\ 4. Department of Medicine, Meharry Medical Center, Nashville, TN, 37208, USA. \\ *first author \\ $\triangle$ Corresponding author: Sang Cheul Oh, MD, PhD, Division of Oncology/Hematology, Department of Internal Medicine, Korea University Guro Hospital, 148 \\ Gurodong-gil, Guro-gu, Seoul 152-703, Korea; Phone: +82 22626 1147; Fax: +82 22626 1148; E-mail: sachoh@korea.ac.kr
}

(c) The author(s). This is an open access article distributed under the terms of the Creative Commons Attribution License (https://creativecommons.org/licenses/by/4.0/). See http://ivyspring.com/terms for full terms and conditions.

Received: 2019.03.11; Accepted: 2019.09.27; Published: 2020.01.01

\begin{abstract}
Oxaliplatin is used for treatment in combination with many drugs. However, the survival rate is still low due to side effects and drug resistance. Therefore, the combination with natural products was required for increasing efficacy and reducing side effects.

Genipin, a natural product derived from the Gardenia jasminoides, associated with anti-angiogenic, anti-proliferative, hypertension, inflammatory, and the Hedgehog pathway. It is not known that genipin increases the therapeutic effect of oxaliplatin in gastric cancer. In this study, we found that genipin sensitizes oxaliplatin-induced apoptosis for the first time using colony forming assay, FACS analysis, and western blotting in gastric cancer. Additionally, genipin induced p53 expression in AGS, MKN45, and MKN28 cells. Also, genipin induced autophagy and LC3 expression. Knockdown of LC3 decreased cell death enhanced by the combination of oxaliplatin and genipin. In summary, we showed that genipin increases the oxaliplatin-induced cell death via p53-DRAM autophagy. Based on this, we suggest that genipin is a sensitizer of oxaliplatin.
\end{abstract}

Key words: Oxaliplatin; Genipin; p53; Autophagy.

\section{Introduction}

Gastric cancer is frequently occurring disease of cancer-related death worldwide [1]. Recently, although the survival rate of gastric cancer patients has increased, it is still low in a 5-year overall survival [2]. The combination chemotherapy such as cisplatin/5-FU, docetaxel/cisplatin/5-FU, and epirubicin/cisplatin/5-FU has been considered as a standard treatment of gastric cancer [3]. However, these treatments are still insufficient due to side effects, resistance, and low efficacy. The combination therapies with other natural products are needed to enhance chemo-efficacy decrease side effects.

Oxaliplatin, as alternative to cisplatin, is a third generation platinum agent. Oxaliplain is forming
platinum-DNA adducts to disrupt DNA transcription and replication, resulting in DNA damage and apoptosis [4, 5]. However, because oxaliplatin has side effects, it is necessary to increase anti-cancer drug efficacy and reduce side effects by combining with natural products.

Genipin, a natural product derived from the Gardenia jasminoides [6], associated with anti-angiogenic, anti-proliferative, hypertension, inflammatory, and the Hedgehog pathway [7-9]. Genipin has anti-cancer effect in various cancers, such as gastric cancer, colorectal cancer, hepatocellular carcinoma [10, 11]. It is not known that genipin increases the therapeutic effect of oxaliplatin in gastric 
cancer.

Autophagy, a conserved degradation process, is associated with catabolic processes, autoimmunity, cellular development, and cell death [12-14]. Autophagy is called type II programmed cell death, unlike apoptosis. However, both apoptosis and autophagy can be induced by similar upstream signaling pathways [15]. As LC3 is a marker of autophagosomal membranes, change of LC3-II expression are associated with the autophagic activity via lysosome [16].

p53, tumor suppressor gene, is known to regulate the $\mathrm{BH} 3$-domain proteins PUMA and NOXA [17]. Moreover, p53 has been shown to play a critical function in autophagy [18, 19]. Damage-regulated autophagy modulator (DRAM), a phylogenetically ancient lysosomal protein that contributes to p53-induced autophagy [20]. Recently, in the previous studies, p53-DRAM induced autophagy has been also shown to enhance cancer therapy by increasing cytotoxicity of breast cancer cells [21].

In this study, we investigated whether genipin sensitizes oxaliplatin-induced apoptosis in gastric cancer. We found that genipin increased p53 expression. Additionally, we found that genipin induced autophagy. In particular, genipin enhanced autophagy through increase of DRAM. Collectively, we suggest that genipin increases the oxaliplatin-induced cell death via p53-DRAM autophagy in gastric cancer.

\section{Materials and methods}

\section{Cell culture}

Human Gastric cancer cell lines, AGS, MKN45, and MKN28 were purchased from the Korea Cell Line Bank. Cells were cultured in RPMI 1640 medium (Invitrogen, Carlsbad, CA, USA) with $10 \%$ fetal bovine serum (Sigma-Aldrich, St. Louis, MO, USA), 1 $\mathrm{mM}$ L-glutamine, and $26 \mathrm{mM}$ sodium bicarbonate for monolayer cell culture. The gastric epithelial cell line, HFE-145 was gifted from Dr. Hassan Ashktorab (Howard University). All cell lines were grown at $37^{\circ} \mathrm{C}$ in a humidified chamber with $5 \% \mathrm{CO}_{2}$.

\section{Reagents and antibodies}

Genipin was purchased from Calbiochem (San Diego, CA, USA). Oxaliplatin was purchased from Sigma-Aldrich (St. Louis, MO, USA). Anti-Bak, anti-Bcl-2, anti-Mcl-1, anti-Bcl-xL, anti-p53, and anti-DRAM antibodies were purchased from Santa Cruz Biotechnology (Santa Cruz, CA, USA). Anti-XIAP, anti-NOXA, anti-BIM, anti-survivin, anti-cleaved PARP, anti-caspase-3, anti-caspase-9, and anti-LC3 were purchased from Cell Signaling
Technology (Danvers, MA, USA). Anti-actin antibody was purchased from Sigma-Aldrich. For the secondary antibodies, anti-mouse IgG HRP and anti-rabbit IgG HRP were purchased from Cell Signaling Technology.

\section{Western blotting}

Cells were lysed in RIPA buffer (50 mM Tris, $150 \mathrm{mM} \mathrm{NaCl}, 1 \%$ Triton X-100, 0.1\% SDS, and 1\% Na deoxycholate $[\mathrm{pH}$ 7.4]) with a protease and phosphatase inhibitor cocktail (Sigma-Aldrich). Protein concentrations were measured using the bicinchoninic acid protein assay reagent (Thermo Fisher Scientific, Waltham, MA, USA). Equal amounts of proteins were separated by SDS-PAGE and transferred to nitrocellulose membranes (GE Healthcare Life Sciences, Little Chalfont, UK). The membranes were blocked with TBS containing $0.2 \%$ Tween 20 and 5\% skim milk, incubated with primary antibodies overnight at $4^{\circ} \mathrm{C}$, and then incubated with HRP-labeled secondary antibodies. The signals were detected by X-ray film.

\section{Flow cytometry analysis of cell apoptosis}

The translocation of phosphatidylserine, an apoptosis marker, from the inner to the outer leaflet of the plasma membrane was detected by the binding of allophycocyanin-conjugated annexin V. Briefly, HCT116 cells untreated or treated with genipin, oxaliplatin, or a combination of the two agents were resuspended in the binding buffer provided with the Annexin V-FITC Apoptosis Detection Kit (BioBud, Cat. No. LS-02-100). Cells were mixed with $1.25 \mu \mathrm{L}$ of the Annexin V- $7 \mu \mathrm{L}$ FITC reagent and incubated for $30 \mathrm{~min}$ at $4^{\circ} \mathrm{C}$ in the dark. The staining was then terminated and cells were immediately analyzed by flow cytometry.

\section{Small interfering RNA (siRNA)}

p53 siRNA, LC3 siRNA, and negative control siRNA were purchased from Santa Cruz Biotechnology. Cells were transfected with siRNA oligonucleotides using Lipofectamine RNAi Max reagents (Invitrogen) according to the manufacturer's instructions.

\section{Autophagy detection}

Cells grown on glass coverslips were treated with genipin for $24 \mathrm{~h}$. After $24 \mathrm{~h}$, the medium removed and washed the cells twice with $1 X$ assay buffer. Cells treated microscopy dual detection reagent $100 \mu \mathrm{L}$ for $30 \mathrm{~min}$ at $37^{\circ} \mathrm{C}$. Cells were washed $1 \mathrm{X}$ assay buffer, followed by fixation $4 \%$ formaldehyde for $20 \mathrm{~min}$. The nuclei were co-stained with DAPI. The cells were mounted in Vectashield mounting medium (Vector laboratories, Burlingame, CA, USA) and visualized by 
fluorescence microscopy.

\section{Immunofluorescence staining}

Cells grown on glass coverslips were fixed with $3.7 \%$ formaldehyde for $15 \mathrm{~min}$, followed by permeabilization with $0.5 \%$ Triton X-100 for $15 \mathrm{~min}$ at room temperature. Cells were then blocked for $1 \mathrm{~h}$ with 3\% bovine serum albumin and probed with primary antibodies overnight at $4^{\circ} \mathrm{C}$, followed by incubation with secondary Alexa fluor-594-conjugated secondary antibody (Molecular Probes, Eugene, OR, USA) or FITC-conjugated secondary antibody (Sigma-Aldrich). The nuclei were co-stained with DAPI. The cells were mounted in Vectashield mounting medium (Vector laboratories, Burlingame, CA, USA) and visualized by fluorescence microscopy.

\section{Combination Index and Statistical analysis}

To determine whether the cytotoxic interactions of genipin and oxaliplatin were synergistic, additive, or antagonistic in gastric cancer cells, drug effects were examined using the combination index (CI) method of Chou and Talalay. GraphPad InStat 6 software was used for all statistical analyses (GraphPad Software, Inc., La Jolla, CA, USA). Statistics were analyzed by using one-way ANOVA with GraphPad InStat 6. One-way ANOVA followed by Turkey post hoc tests were performed in all statistical analysis. To determine the significance between two groups, an unpaired $t$ test was used, where a $p$-value of less than 0.05 was considered significant.

\section{Results}

\section{Genipin enhances oxaliplatin-induced cell death in gastric cancer cell lines}

We first performed an MTT assay to detect cell death induced by oxaliplatin. Oxaliplatin increased cell death in gastric cancer cells (Fig. 1A). To investigate whether genipin increased oxaliplatin-induced cell death, we performed an MTT assay. Genipin induced cell death in gastric cancer cells (Fig 1B and Fig 1C). We examined a combination index (CI) for selecting the most effective concentration using the Compusyn software. As shown in Fig 1E, the combination of $10 \mu \mathrm{M}$ oxaliplatin and $100 \mu \mathrm{M}$ genipin showed the best combination effect (Fig 1E). Next, we examined cell death using trypan blue staining. The combination of oxaliplatin and genipin increased cell death in AGS, MKN45, and MKN28 (Fig 1F), but not in gastric epithelial cell (HFE-145) (Fig 1D). These results indicated that genipin enhanced the sensitivity of oxaliplatin in gastric cancer.

\section{The combination of oxaliplatin and genipin induces apoptosis}

The combination effects of oxaliplatin and genipin on AGS cells morphology was observed under a light microscope (Fig 2A). Colony forming ability was decreased in the combination of oxaliplatin and genipin, compared to oxaliplatin or genipin alone (Fig 2B). To determine whether the effect of combination induced apoptosis, we performed Annexin V/ PI staining with FACS analysis. The combination of oxaliplatin and genipin increased significantly apoptosis (Fig 2C). Additionally, we examined the activation of cleaved poly (ADP-ribose) polymerase (PARP), caspase-9, and caspase- 3 . The combination of oxaliplatin and genipin increased the cleaved forms of these proteins (Fig 2D). The activity of caspase 3/7 was increased in the combination of oxaliplatin and genipin (Fig 2E). To investigate whether the combination effects was depended on caspase, we pretrested z-VAD-fmk, a pan-caspase inhibitor. As expected, z-VAD-fmk inhibited the cleaved forms of PARP, caspase-9, and caspase-3 induced by the combination (Fig 2F).

\section{Genipin enhances oxaliplatin-induced cell death via upregulating p53}

To determine how genipin increased sensitivity of oxaliplatin, we confirmed pro-apoptotic proteins and anti-apoptotic proteins. As shown in Fig 3A, genipin increased expression of p53. Upregulation of p53 by genipin was also confirmed in MKN45 cells (Fig 3B). Additionally, increase of p53 by genipin was confirmed by immunofluorescence (Fig 3C). To verify whether upregulation of p53 by genipin enhanced sensitivity of oxaliplatin, we inhibited p53 using p53 siRNA. We found that the combination effect was decreased by p53 siRNA (Fig 3D). Knockdown of p53 significantly decreased apoptosis using FACS analysis (Fig 3E). These results suggest that genipin enhanced oxaliplain-induced apoptosis through upregulation of p53.

\section{Genipin increases oxaliplatin-induced cell death via autophagy}

As shown in Fig 4A, autophagic morphology was appeared when AGS cells were treated with genipin. To determine whether genipin induced autophagy, we performed autophagy staining. Genipin induced autophagy compared to control (Fig 4B). We also confirmed autophagy-related proteins such as Beclin1, p62, and LC3. As shown in Fig 4C, genipin increased LC3 expression. Additionally, genipin increased DRAM. These indicated that genipin induced autophagy via p53-DRAM pathway. Consistent with protein level, genipin increased LC3 
puncta using immunofluorescence (Fig 4D). To further confirm whether the combination effect of oxaliplatin and genipin is LC3-dependent, we silenced LC3 using LC3 siRNA. LC3 knockdown decreased cell death induced by the combination of oxaliplatin and genipin (Fig 4E). Additionally, LC3 knockdown significantly decreased apoptosis by FACS analysis (Fig 4F). These results suggest that genipin increases sensitivity of oxaliplatin by inducing autophagy (p53-DRAM).

A

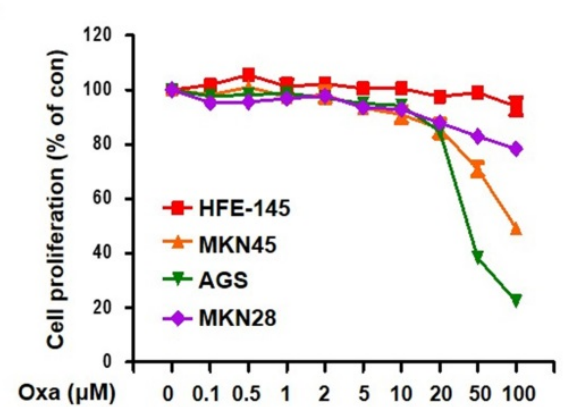

C

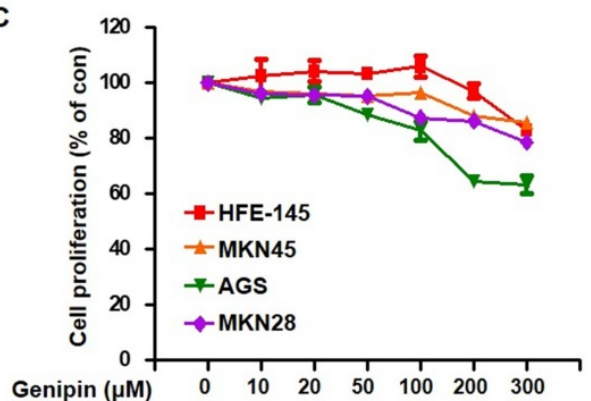

\section{Discussion}

Oxaliplatin is widely used by combination with other drugs such as 5-FU or folinic acid. However, drug resistance and side effects is still a problem. For this problem, we must overcome these by combination with natural products that can increase the effect and reduce side effects. We found that sensitivity of oxaliplatin was increased through the combination with genipin for the first time in gastric cancer.

B<smiles>COC(=O)C1=CO[C@@H](O)[C@H]2CC=C(CO)[C@H]12</smiles>

D

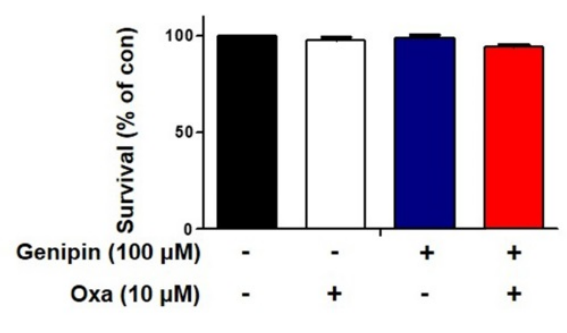

E

\begin{tabular}{c|c|c|c|c}
\hline $\begin{array}{c}\text { Combination } \\
\text { index }\end{array}$ & $\mathbf{0 . 4 4 8 7 1}$ & $\mathbf{0 . 4 4 0 0 5}$ & $\mathbf{0 . 4 5 9 1 2}$ & $\mathbf{0 . 2 9 0 3 5}$ \\
\hline $\begin{array}{c}\text { Description } \\
\text { Oxaliplatin }(\mu \mathrm{M})\end{array}$ & synergism & synergism & synergism & $\begin{array}{c}\text { Strong } \\
\text { synergism }\end{array}$ \\
\hline Genipin $(\mu \mathrm{M})$ & 5 & 5 & 10 & 10 \\
\hline
\end{tabular}

$\mathrm{F}$
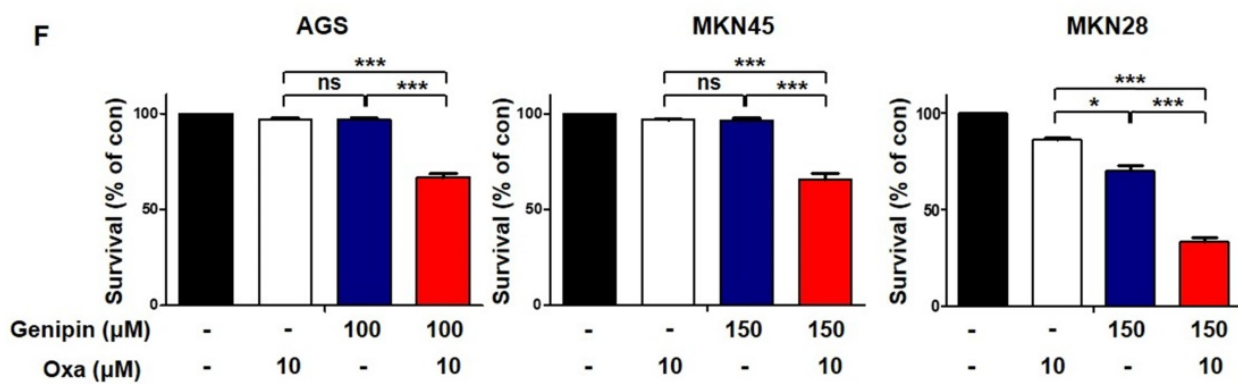

Fig. 1. Genipin enhances oxaliplatin-induced cell death in gastric cancer cell lines. (A) Treatment with $0-100 \mu M$ oxaliplatin for $24 \mathrm{~h}$ in gastric cancer cell lines and HFE-145. (B) Molecular structure of genipin. (C) Cell proliferation of gastric cancer cell lines was measured by using the MTT assay after treatment with 0 $300 \mu \mathrm{M}$ genipin for $24 \mathrm{~h}$ (D) Cell survival of gastric epithelial cell line HFE-145 was measured using trypan blue staining. (E) Combination index for combination of genipin and oxaliplatin, $\mathrm{Cl}=0.1-0.3$, Strong Synergism; $\mathrm{Cl}=0.3-0.7$, Synergism. $(\mathrm{F})$ Cell survival of gastric cancer cells were measured using trypan blue staining. The data are expressed as the mean of three independent experiments. $* * * P<0.001$, $* P<0.05$, ns : not significant. 


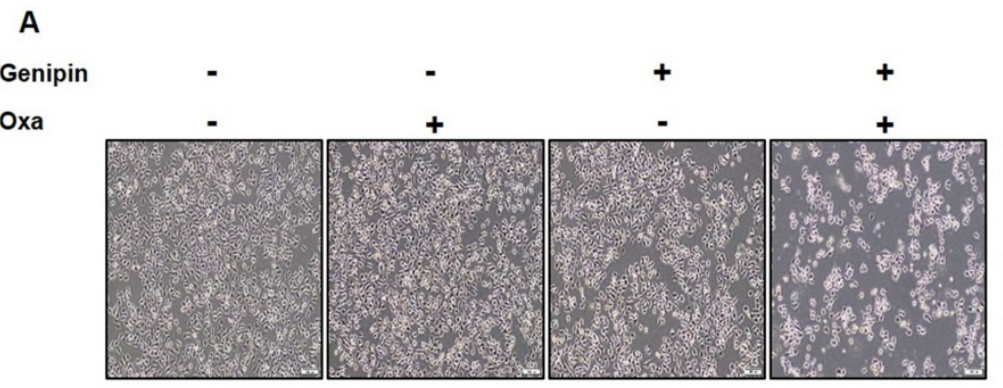

B

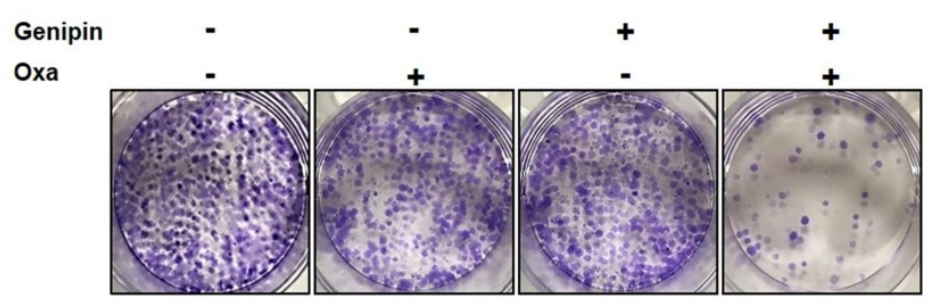

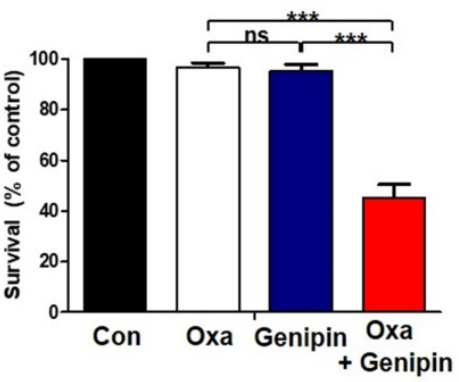

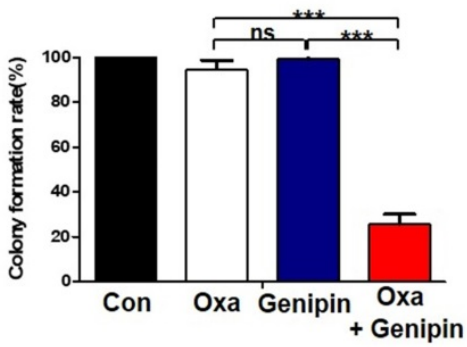

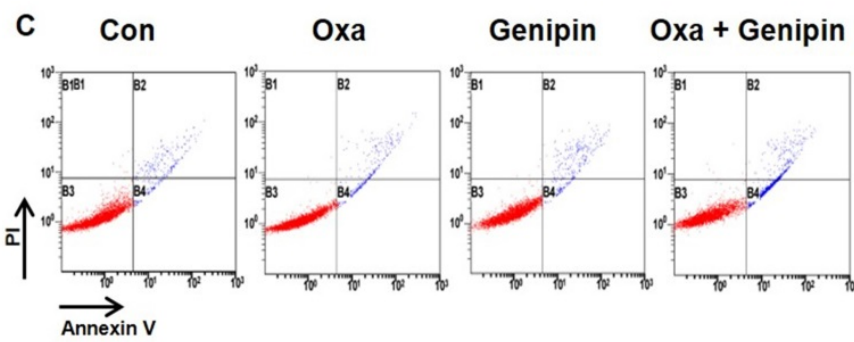

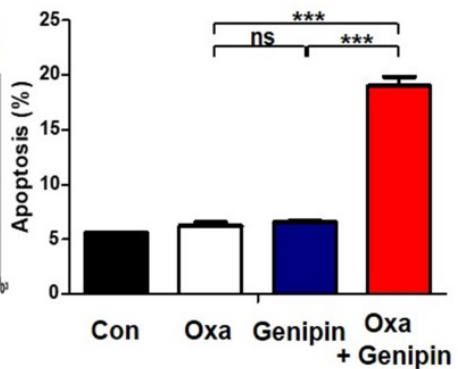

D

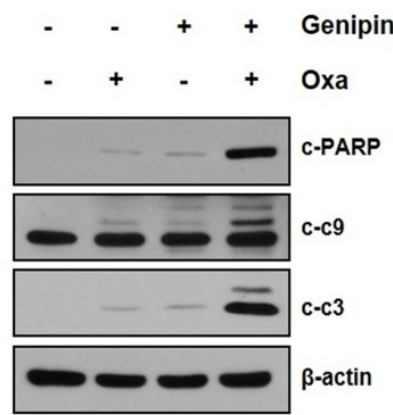

E

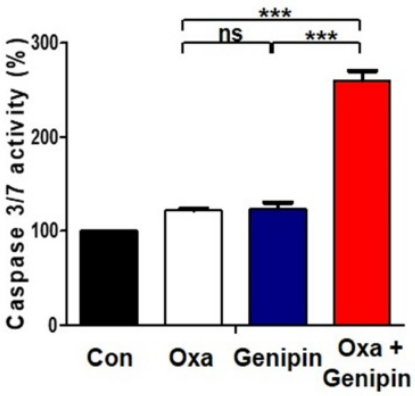

$\mathbf{F}$

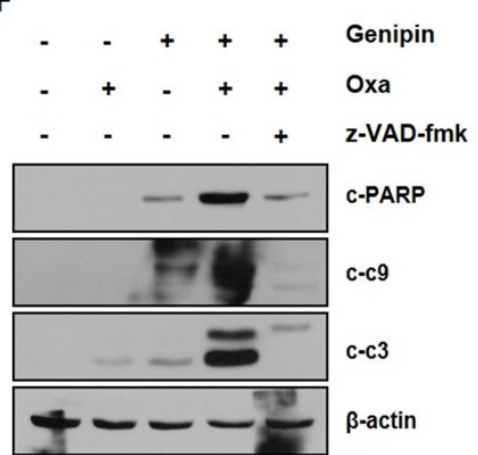

Fig. 2. The combination of oxaliplatin and genipin induces apoptosis. (A) AGS cells were treated with genipin, oxaliplatin, or combination for 24h. The cells were observed by light microscopy. Scale bar: $100 \mu \mathrm{m}$. (B) AGS cells were treated with genipin, oxaliplatin, or combination for $24 \mathrm{~h}$. After 10 days, the cells were stained with crystal violet dye, and photographs of the colonies were obtained. (C) AGS cells were treated with genipin, oxaliplatin, or combination for 24h. The cells were stained with annexin $\mathrm{V}$ and $\mathrm{PI}$ and then were measured using FACS analysis. (D) The activity of cleaved-PARP and cleaved-caspase 3, and cleaved-caspase 9 were measured by western blotting. $\beta$-Actin was used as a loading control for each lane. (E) AGS cells were treated with genipin, oxaliplatin, or combination for $24 \mathrm{~h}$. Caspase 3/7 activity was measured. (F) AGS cells were pretreated with with $25 \mu \mathrm{M}$ z-VAD-fmk for $30 \mathrm{~min}$ and then treated with oxaliplatin, genipin, or combination. The activity of cleaved-PARP and cleaved-caspase 3 , and cleaved-caspase 9 were measured by western blotting. $\beta$-Actin was used as a loading control for each lane. The data are expressed as the mean of three independent experiments. $* * * P<0.001, \mathrm{~ns}:$ not significant.

Our previous study, we found that genipin enhanced oxaliplatin-induced apoptosis in colorectal cancer [22]. In our study, we investigated whether genipin enhanced oxaliplatin induced cell death for gastric cancer. As shown in Fig 1, the combination of oxaliplatin and genipin increased cell death in AGS, MKN45, and MKN28. Additionally, the effect of combination these was confirmed using colony-forming assay, FACS analysis, and western blotting (Fig 2). Our results also showed that p53 is important factor for oxaliplatin sensitivity. Knockdown of p53 decreased genipin-induced oxaliplatin cell death (Fig 3D and Fig 3E).

Autophagy is closely related to cell survival 
pathway in eukaryotes. It associated with the degradation of cellular components such as long-lived proteins, damaged organelles, protein aggregates, and intracellular pathogens [23]. As shown in Fig 4A, we observed autophagic morphology. We also confirmed autophagy induction using autophagy detection kit (Fig 4B). Because genipin increased p53 expression, we confirmed autophagy factors associated with p53 pathway. Genipin significantly increased DRAM expression (Fig 4C). In the previous studies, cytoplasmic p53 is known to suppress autophagy through the activation of mTOR signaling and the inactivation of AMP kinase, whereas nuclear p53 activates autophagy by activation of DRAM which enhances the formation of autophagolysosomes [20, 24]. Knockdown of LC3 decreased genipin-induced oxaliplatin cell death (Fig 4E and Fig 4F).

The connection between autophagy and apoptosis is still controversial. It is not yet clear whether autophagy inhibits apoptosis or whether autophagy activates apoptosis, but both cause cell death through by similar upstream signaling pathways [15]. In this study, we suggest that genipin enhances oxaliplatin-induced cell death via p53-apoptosis pathway and p53-DRAM-autophagy pathway (Fig 4G).
A

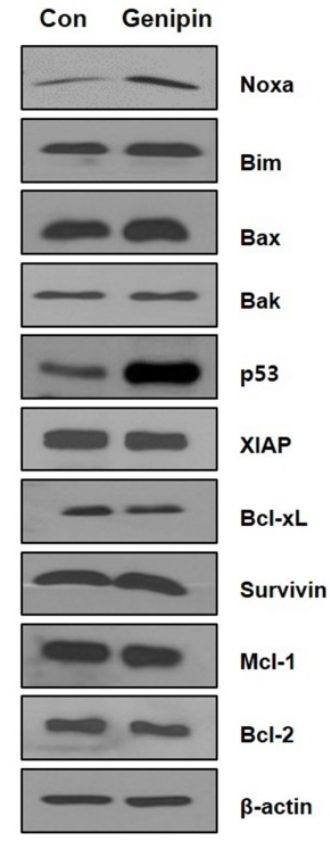

D
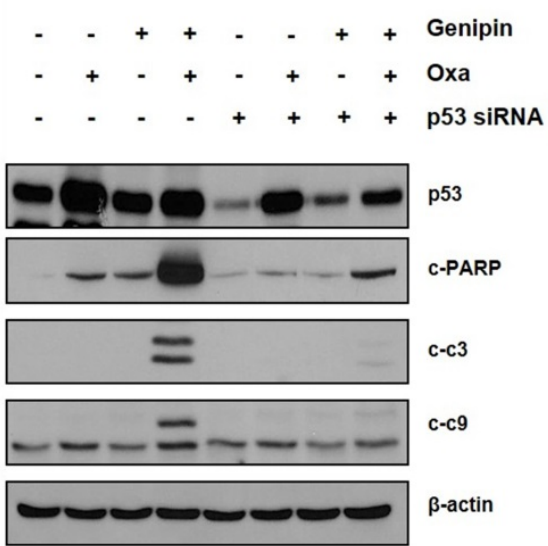

B

c
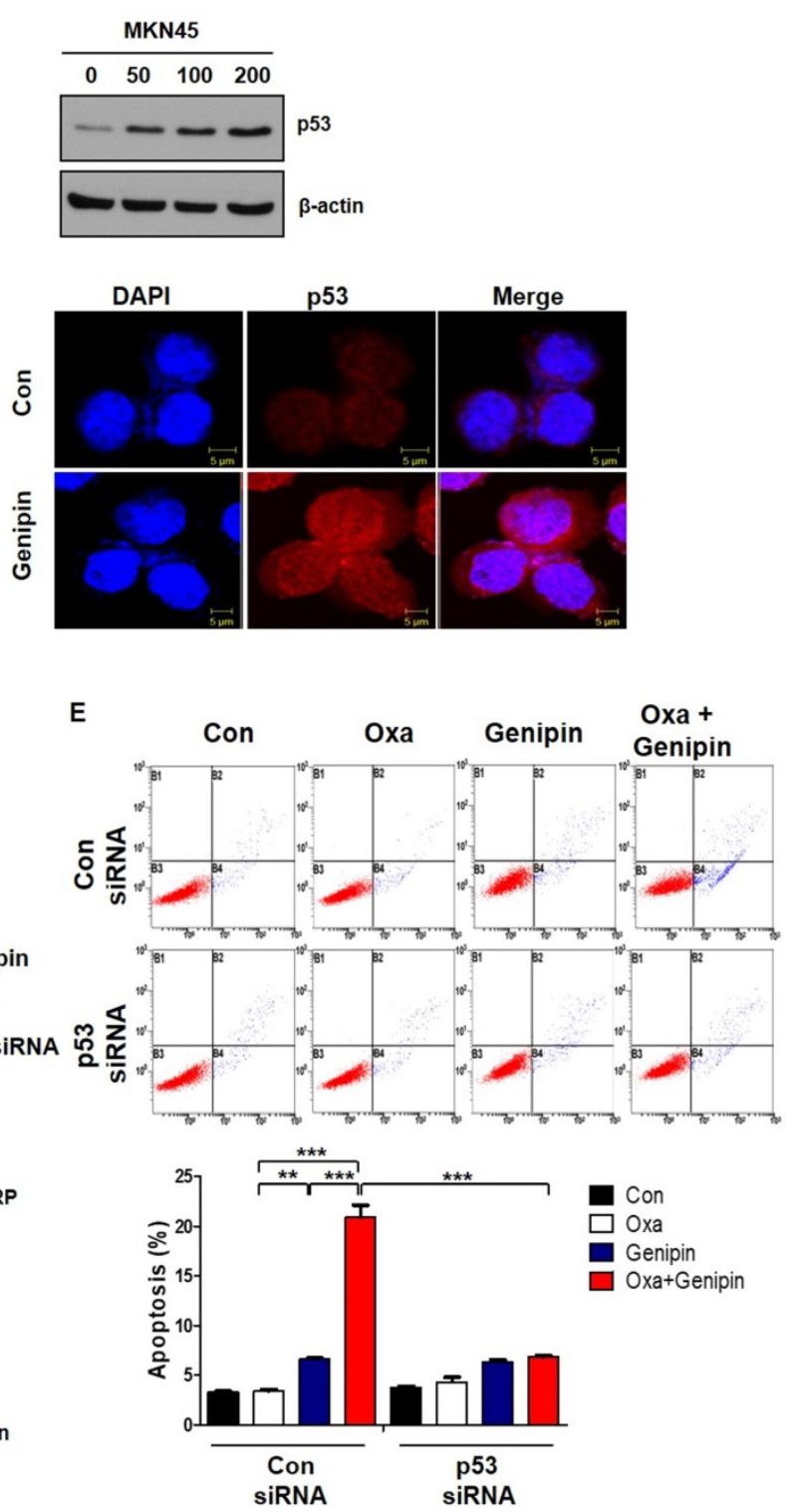

Fig. 3. Genipin enhances oxaliplatin-induced cell death via upregulating p53. (A) AGS cells were treated with genipin $100 \mu M$ for $24 \mathrm{~h}$. The apoptotic proteins and anti-apoptotic proteins were measured by western blotting. $\beta$-Actin was used as a loading control for each lane. (B) MKN45 cells were treated genipin for 24h. The protein expression of $\mathrm{p} 53$ were measured by western blotting. (C) Immunofluorescence of $\mathrm{p} 53$ was detected by confocal laser-scanning microscopy (original magnification: 40x). Scale bar: $5 \mu$ M (D) AGS cells were transfected with control siRNA or p53 siRNA and then the cells were treated with oxaliplatin, genipin, or combination. The activity of cleaved-PARP and cleaved-caspase 3, and cleaved-caspase 9 were measured by western blotting. (E) AGS cells were transfected with control siRNA or p53 siRNA and then the cells were treated with oxaliplatin, genipin, or combination. The cells were stained with annexin $\mathrm{V}$ and PI and then were measured using FACS analysis. $* * * P<0.001, * * P<0.01$. 


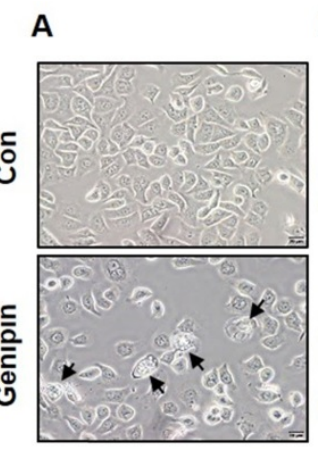

B

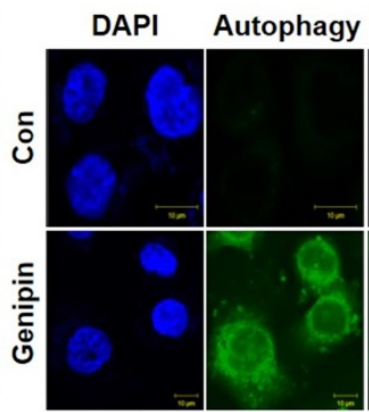

D
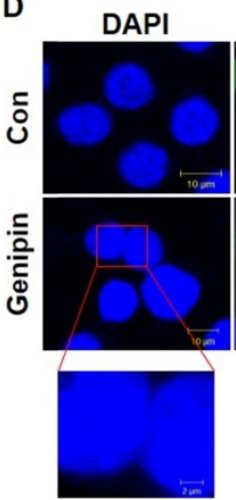

F

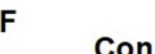

LC3

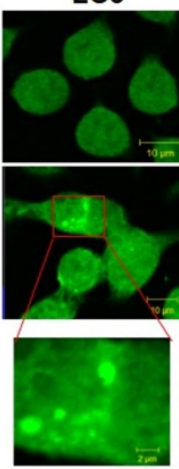

Con
Merge

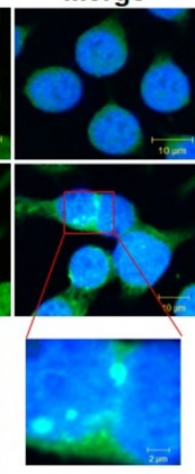

Oxa +
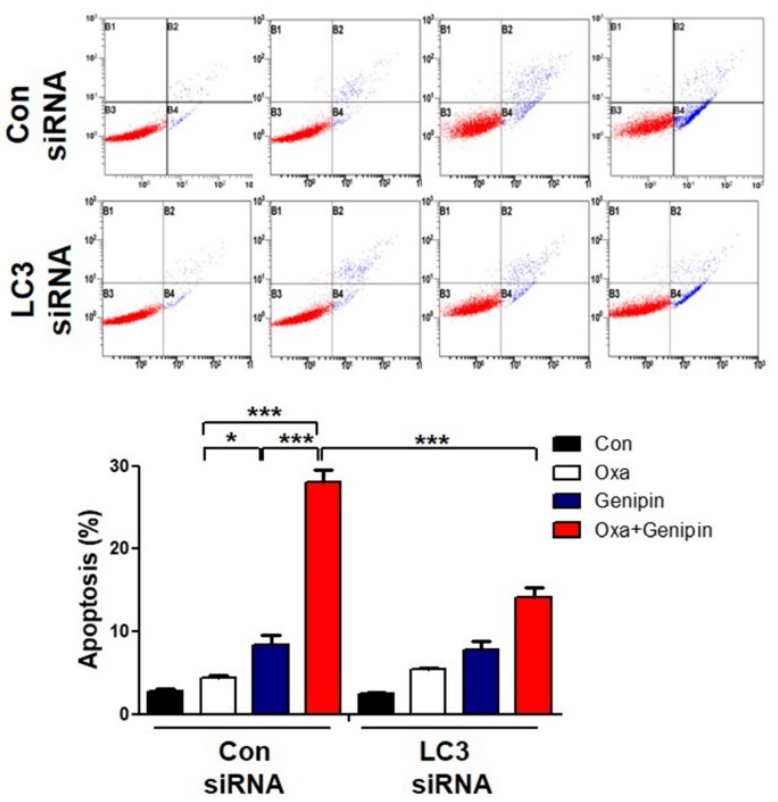

C

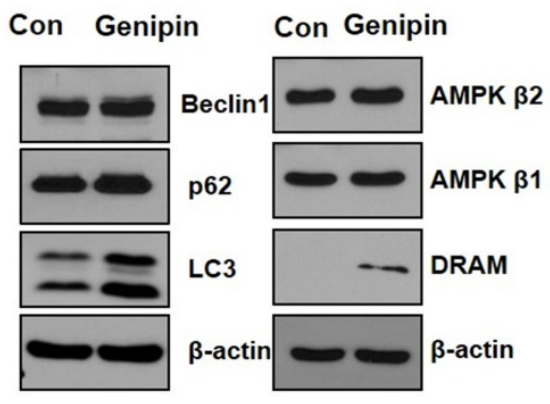

E
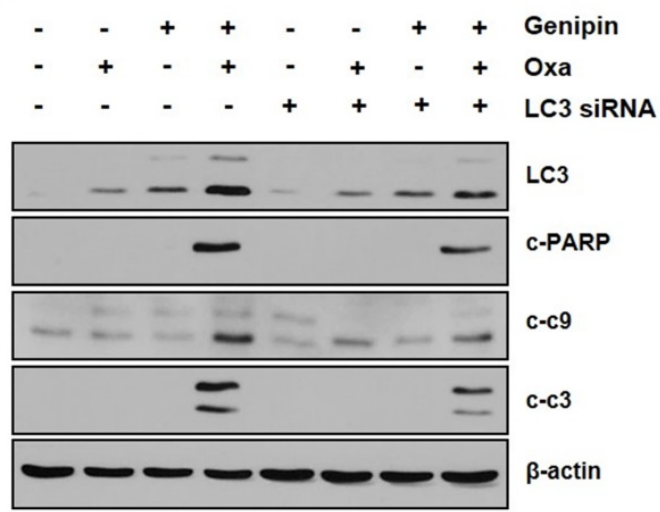

LC3

C-PARP

c-c9

c-c3

$\beta$-actin
G
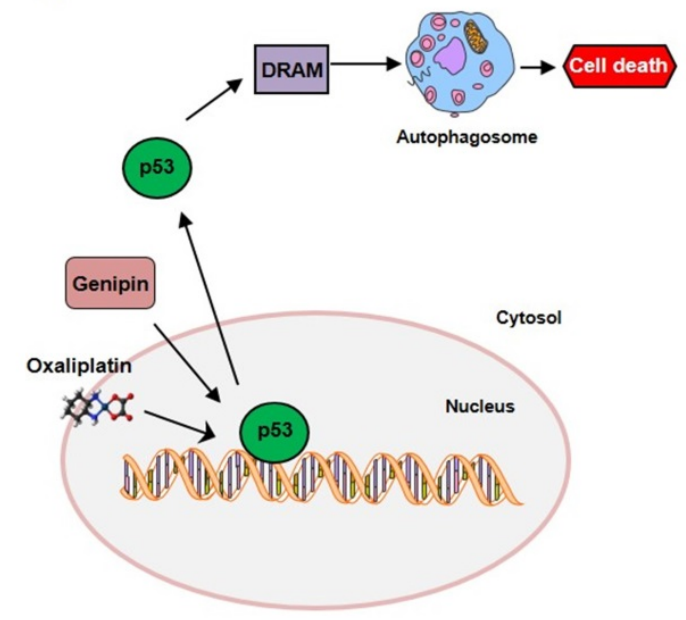

Fig. 4. Genipin increases oxaliplatin-induced cell death via autophagy. (A) AGS cells were treated with genipin $100 \mu M$ for $24 h$. The cells were observed by light microscopy. Scale bar: $20 \mu \mathrm{m}$. (B) The autophagy was observed by immunofluorescence using autophagy detection kit (original magnification: $40 \times$ ). Scale bar: 10 $\mu M$. (C) AGS cells were treated with genipin $100 \mu \mathrm{M}$ for $24 \mathrm{~h}$. The protein expression of Beclin 1, P62, LC 3 , AMPK $\beta 1$, AMPK $\beta 2$, and DRAM were measured by western blotting. $\beta$-Actin was used as a loading control for each lane. (D) The LC3 puncta were observed by immunofluorescence (original magnification: $40 \times$ ). Scale bar: $10 \mu \mathrm{M}$. (E) AGS cells were transfected with control siRNA or LC3 siRNA and then the cells were treated with oxaliplatin, genipin, or combination. The activity of cleaved-PARP and cleaved-caspase 3, and cleaved-caspase 9 were measured by western blotting. (F) AGS cells were transfected with control siRNA or LC3 siRNA and then the cells were treated with oxaliplatin, genipin, or combination. The cells were stained with annexin $\mathrm{V}$ and PI and then were measured using FACS analysis. (G) Schematic diagram for combination model of oxaliplatin and genipin. $* * * P<0.001, * P<0.05$.

Recently, combination therapies with more than 30 natural products have been undergo in clinical trials for cancer treatment, but studies on the combined effects of genipin and oxaliplatin are also needed in gastric cancer patients-derived cells.

Taken together, our study can be the baseline research for future clinical trials and suggests genipin as a novel sensitiser of oxaliplatin. 


\section{Abbreviations}

PARP: poly (ADP-ribose) polymerase; PBS: phosphate-buffered saline solution; SDS-PAGE: sodium dodecyl sulfate polyacrylamide gel electrophoresis; PMSF: phenylmethylsulfonyl fluoride; TBS: Tris-buffered saline; DAPI: 4,6-diamidino-2-phenylindole; siRNA: short interfering RNA; HRP: horseradish peroxidase; RIPA: radioimmunoprecipitation assay; TBS: Tris-buffered saline; FITC: fluorescein isothiocyanate; PMSF: phenylmethylsulfonyl fluoride.

\section{Acknowledgements}

This work was supported by the National Research Foundation (NRF) of Korea grant funded by the Korean government (MSIP) [NRF-2017R1A6 A3A11030765] and [NRF-2017R1A2B2011684] and supported by a Korea University Grant.

\section{Authors' contributions}

BRK conceived and designed the study, provided financial support, collected and assembled the data, analyzed and interpreted the data, and wrote the manuscript. YAJ, DYK, and JLK conceived and designed the study and analyzed and interpreted the data. SYJ and DHL conceived and designed the study. YJN, SHP, MJJ, HKY collected and assembled the data, and analyzed and interpreted the data. SCO and conceived and designed the study, provided financial support, collected and assembled the data, analyzed and interpreted the data, wrote the manuscript, and provided final approval of manuscript. All authors discussed the results and commented on the manuscript.

\section{Ethics approval and consent to participate}

All experiments were approved by the Ethics Committee of Korea University.

\section{Competing Interests}

The authors have declared that no competing interest exists.

\section{References}

1. Carlomagno N, Incollingo P, Tammaro V, Peluso G, Rupealta N, Chiacchio G, et al. Diagnostic, Predictive, Prognostic, and Therapeutic Molecular Biomarkers in Third Millennium: A Breakthrough in Gastric Cancer. Biomed Res Int. 2017; 2017: 7869802.

2. Charalampakis N, Economopoulou P, Kotsantis I, Tolia M, Schizas D, Liakakos T, et al. Medical management of gastric cancer: a 2017 update. Cancer Med. 2018; 7: 123-33.

3. Morabito A, Carillio G, Longo R. Systemic treatment of gastric cancer. Crit Rev Oncol Hematol. 2009; 70: 216-34.

4. Kelland L. The resurgence of platinum-based cancer chemotherapy. Nat Rev Cancer. 2007; 7: 573-84

5. Hato SV, Khong A, de Vries IJ, Lesterhuis WJ. Molecular pathways: the immunogenic effects of platinum-based chemotherapeutics. Clin Cancer Res. 2014; 20: 2831-7.

6. Muzzarelli RA, El Mehtedi M, Bottegoni C, Gigante A. Physical properties imparted by genipin to chitosan for tissue regeneration with human stem cells: A review. Int J Biol Macromol. 2016; 93: 1366-81.
7. Nam KN, Choi YS, Jung HJ, Park GH, Park JM, Moon SK, et al. Genipin inhibits the inflammatory response of rat brain microglial cells. Int Immunopharmacol. 2010; 10: 493-9.

8. Feng Q, Cao HL, Xu W, Li XR, Ren YQ, Du LF. Apoptosis induced by genipin in human leukemia K562 cells: involvement of c-Jun N-terminal kinase in G(2)/M arrest. Acta Pharmacol Sin. 2011; 32: 519-27.

9. Kim BR, Jeong YA, Na YJ, Park SH, Jo MJ, Kim JL, et al. Genipin suppresses colorectal cancer cells by inhibiting the Sonic Hedgehog pathway. Oncotarget. 2017; 8: 101952-64.

10. Ko H, Kim JM, Kim SJ, Shim SH, Ha CH, Chang HI. Induction of apoptosis by genipin inhibits cell proliferation in AGS human gastric cancer cells via Egr1/p21 signaling pathway. Bioorg Med Chem Lett. 2015; 25: 4191-6.

11. Ye J, Li J, Wang X, Li L. Medicinal supplement genipin induces p53 and Bax-dependent apoptosis in colon cancer cells. Oncol Lett. 2018; 16: 2957-64.

12. Yue $Z$, Jin $S$, Yang $C$, Levine AJ, Heintz N. Beclin 1, an autophagy gene essential for early embryonic development, is a haploinsufficient tumor suppressor. Proc Natl Acad Sci U S A. 2003; 100: 15077-82.

13. Kroemer G, Levine B. Autophagic cell death: the story of a misnomer. Nat Rev Mol Cell Biol. 2008; 9: 1004-10.

14. Deretic V, Delgado M, Vergne I, Master S, De Haro S, Ponpuak M, et al. Autophagy in immunity against mycobacterium tuberculosis: a model system to dissect immunological roles of autophagy. Curr Top Microbiol Immunol. 2009; 335: 169-88.

15. Eisenberg-Lerner A, Bialik S, Simon HU, Kimchi A. Life and death partners: apoptosis, autophagy and the cross-talk between them. Cell Death Differ. 2009; 16: 966-75.

16. Tanida I, Minematsu-Ikeguchi N, Ueno T, Kominami E. Lysosomal turnover, but not a cellular level, of endogenous LC3 is a marker for autophagy. Autophagy. 2005; 1: 84-91.

17. Riley $\mathrm{T}$, Sontag E, Chen P, Levine A. Transcriptional control of human p53-regulated genes. Nat Rev Mol Cell Biol. 2008; 9: 402-12.

18. White E. Autophagy and p53. Cold Spring Harb Perspect Med. 2016; 6: a026120.

19. Maiuri MC, Galluzzi L, Morselli E, Kepp O, Malik SA, Kroemer G. Autophagy regulation by p53. Curr Opin Cell Biol. 2010; 22: 181-5.

20. Crighton D, Wilkinson S, O'Prey J, Syed N, Smith P, Harrison PR, et al. DRAM, a p53-induced modulator of autophagy, is critical for apoptosis. Cell. 2006; 126: $121-34$

21. Kim TH, Kim HS, Kang YJ, Yoon S, Lee J, Choi WS, et al. Psammaplin A induces Sirtuin 1-dependent autophagic cell death in doxorubicin-resistant MCF-7/adr human breast cancer cells and xenografts. Biochim Biophys Acta. 2015; 1850: 401-10.

22. Kim BR, Jeong YA, Jo MJ, Park SH, Na YJ, Kim JL, et al. Genipin Enhances the Therapeutic Effects of Oxaliplatin by Upregulating BIM in Colorectal Cancer. Mol Cancer Ther. 2019; 18: 751-61.

23. Levine B, Klionsky DJ. Development by self-digestion: molecular mechanisms and biological functions of autophagy. Dev Cell. 2004; 6: 463-77.

24. Feng Z. p53 regulation of the IGF-1/AKT/mTOR pathways and the endosomal compartment. Cold Spring Harb Perspect Biol. 2010; 2: a001057. 